Draft \#1

\title{
Controlling cell motion and microscale flow with polarized light fields
}

\author{
Siyuan Yang, ${ }^{1}$ Mingji Huang, ${ }^{1}$ Yongfeng Zhao, ${ }^{1}$ and H. P. Zhang ${ }^{1,2, *}$ \\ ${ }^{1}$ School of Physics and Astronomy and Institute of Natural Sciences, \\ Shanghai Jiao Tong University, Shanghai 200240, China \\ ${ }^{2}$ Collaborative Innovation Center of Advanced Microstructures, Nanjing 210093, China
}

(Dated: February 9, 2021)

\begin{abstract}
We investigate how light polarization affects the motion of photo-responsive algae, Euglena gracilis. In a uniformly polarized field, cells swim approximately perpendicular to the polarization direction and form a nematic state with zero mean velocity. When light polarization varies spatially, cell motion is modulated by local polarization. In such light fields, cells exhibit complex spatial distribution and motion patterns which are controlled by topological properties of the underlying fields; we further show that ordered cell swimming can generate directed transporting fluid flow. Experimental results are quantitatively reproduced by an active Brownian particle model in which particle motion direction is nematically coupled to local light polarization.
\end{abstract}


Natural microswimmers, such as bacteria and algae, can achieve autonomous motion by converting locally stored energy into mechanical work [1-15]. Such cellular motility is not only an essential aspect of life but also an inspirational source to develop artificial microswimmers, which propel themselves through self-generated fields of temperature, chemical concentration, or electric potential [1, 3, 7, 12, 13]. Both natural and artificial microswimmers have been used in a wide variety of applications [16-19].

To properly function in a fluctuating heterogeneous environment, microswimmers need to adjust their motility in response to external stimuli [20 23]. For example, intensity and direction of ambient light can induce a variety of motility responses in photosynthetic microorganisms [24 38] and artificial microwimmers [39 45]; these responses have been frequently used to control microswimmer motion [27, 30, 34, 36, 45, 53]. Besides intensity and direction, light polarization can also affect microswimmer motility and lead to polarotaxis: Euglena gracilis cells align their motion direction perpendicular to the light polarization, possibly to maximize the light absorption [54, 55]; artificial microswimmers consisting of two dichroic nanomotors move in the polarization direction [44]. These previous experiments have focused on uniform light fields [44, 54, 55]. The possibility to use complex polarization patterns to control polarotactic microswimmers has not been explored.

In this letter, we investigate Euglena gracilis cell motion in various polarized light fields in a quantitative and systematic fashion. Our experiments show that while spatially uniform polarization aligns cells into a global nematic state with no net motion, spatially varying fields can induce both local nematic order and mean cell motion. Further, we show that ordered cell swimming motion generates fluid flow that can transport passive tracers. Using the experimental data of individual cells, we construct a model to describe the influence of local light polarization on cell orientation dynamics and quantitatively reproduce all experimental observations.

Experiments - Euglena gracilis are unicellular flagellated microorganisms with a rodshaped body of a length $\sim 50 \mu \mathrm{m}$ and a width $\sim 5 \mu \mathrm{m}$. As shown in Fig. 1(a) and Movie S1 in the Supplemental Material [56], cells swim at a mean speed $\sim 60 \mu \mathrm{m} / \mathrm{s}$ (with a standard deviation of $10 \mu \mathrm{m} / \mathrm{s}$.), while rolling around their long axis at a frequency of $1-2 \mathrm{~Hz}$ [57]. A photoreceptor on Euglena cell surface, marked as a red dot in Fig. 1(b), senses surrounding light and generate signals to modulate flagellar beating pattern [33, 58].

In our experiments, Euglena culture is sealed in a disk-shaped chamber $(\sim 150 \mu \mathrm{m}$ in 


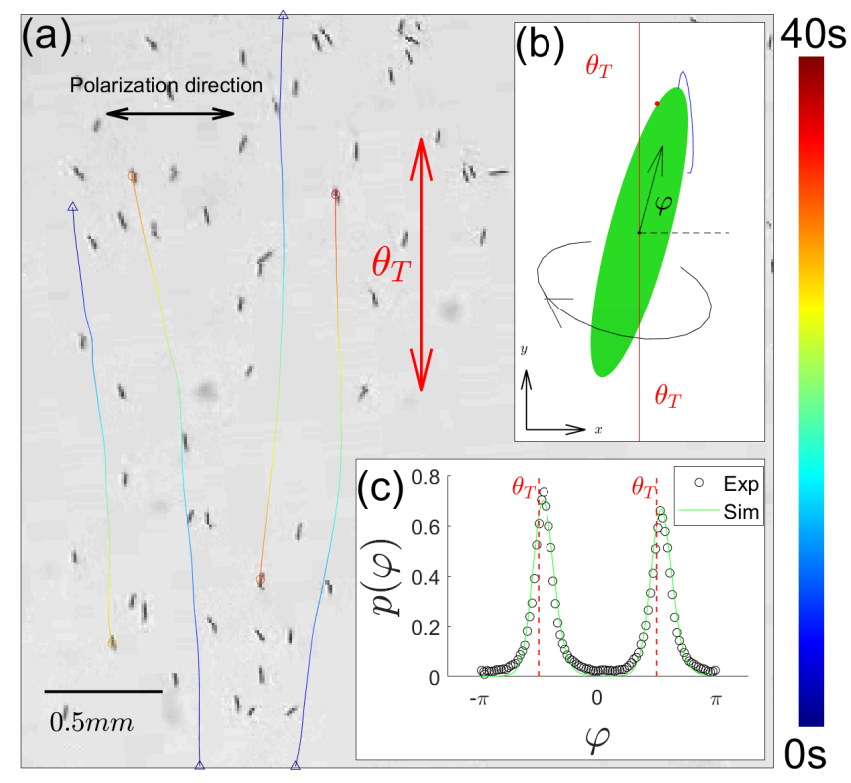

Figure 1. Cell motion in a uniformly polarized light field. (a) Cell trajectories (color-coded by time) plotted on an experimental snapshot. Light polarization is horizontal and cells tend to swim vertically in the targeted direction $\theta_{T}$. (b) shows a schematic for a cell (with a red eye-spot and a flagellum) which moves at a $\varphi$ direction; a circular arrow indicates body rolling motion. (c) Probability distribution of cell motion direction $\varphi$.

thickness and $24 \mathrm{~mm}$ in diameter), which is placed in an illuminating light path, as shown in Fig. S1 [56]. A collimated blue light beam is used to excite cell photo-responses; the default light intensity is $100 \mu \mathrm{W} / \mathrm{cm}^{2}$. Various polarized optical fields can be generated by using different birefringent liquid crystal plates and by changing relative angles between optical elements [59]. Cell motion is recorded by a camera mounted on a Macro-lens. Default system cell density ( $\rho_{0}=8$ cells $/ \mathrm{mm}^{2}$ ) is sufficiently low that we can use a standard particle tracking algorithm [60] to measure position, orientation, and velocity of cells. The current work mainly focuses on steady state dynamics that is invariant over time.

Uniformly polarized light field - Euglena photoreceptor contains dichroically oriented chromoproteins which lead to polarization-dependent photo responses [33, 54, 55, 61]. As shown in Fig. 1(a), cells in a horizontally polarized field tend to orient and swim perpendicularly to the polarization [54]; we denote such a targeted direction for cells as $\theta_{T}$. Quantitatively, we measure the $j$ th cell's location $\vec{r}_{j}(t)$, velocity $\vec{v}_{j}$, and velocity angle $\varphi_{j}$, cf. Fig. 1(b). 

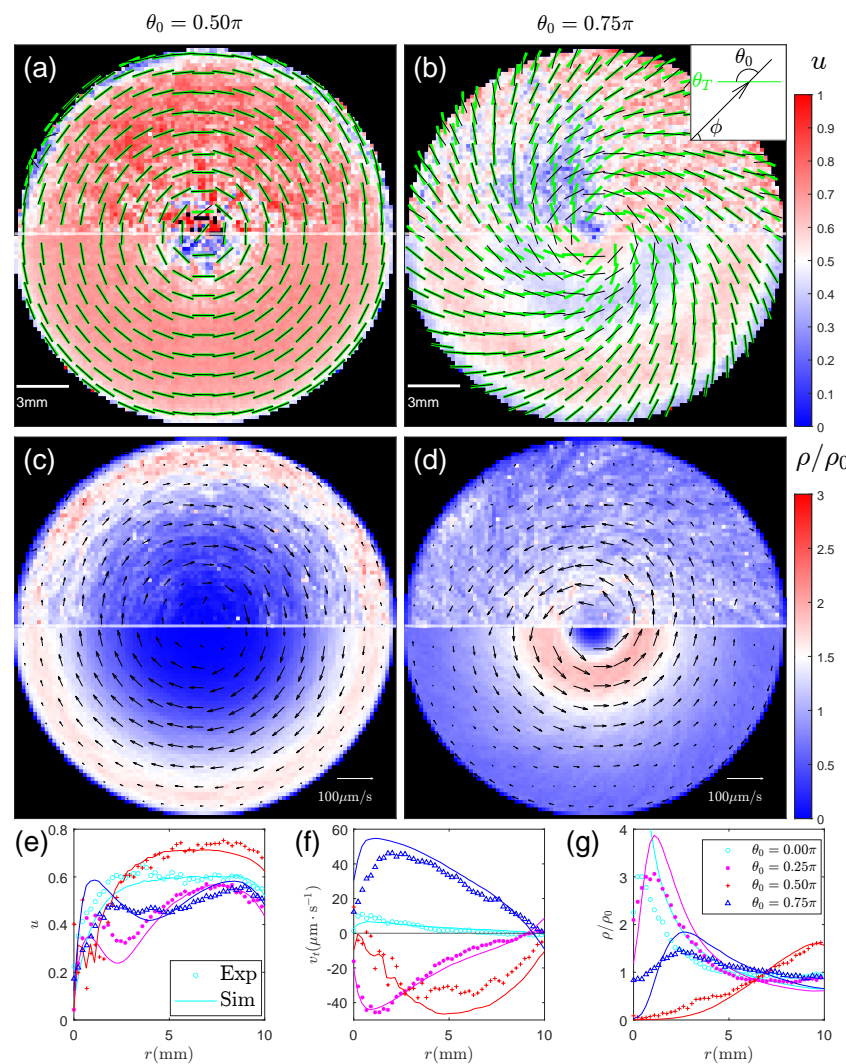

Figure 2. Orientation, velocity, and cell density in axisymmetric light fields containing a $k=+1$ defect with $\theta_{0}=\pi / 2(\mathrm{a}, \mathrm{c})$ and $\theta_{0}=3 \pi / 4(\mathrm{~b}, \mathrm{~d})$. In (a-b), targeted direction $\theta_{T}$ and mean cell motion direction $\phi_{u}$ are shown by green and black lines, respectively, on nematic order parameter $u$ (in color). In (c-d), mean cell velocity $\vec{v}$ is plotted on mean density (in color). In (a-d), top and bottom halves (separated by a white line) are experimental and numerical results, respectively. The inset of (b) defines three angles (see text). (e-g) Radial profiles of nematic order parameter $u$, tangential velocity $v_{t}=\vec{v} \cdot \hat{\phi}$, and cell density $\rho$ for four fields.

Over a square window $\left(1.2 \mathrm{~mm}^{2}\right)$, we define mean cell velocity as $\vec{v}=\left\langle\vec{v}_{j}\right\rangle$, where average $\langle\cdot\rangle$ runs over all cells in the region during the measurement time; nematic order parameter and orientation angle are defined as $u=\left|\left\langle\exp \left(\mathrm{i}\left(2 \varphi_{j}\right)\right)\right\rangle\right|$ and $\phi_{u}=\frac{1}{2} \operatorname{Arg}\left(\left\langle\exp \left(\mathrm{i}\left(2 \varphi_{j}\right)\right)\right\rangle\right)$, where Arg denotes the phase angle of a complex number. In uniform fields, cells are homogeneously distributed over space and form a global nematic state with a vanishing mean cell velocity: $u \approx 0.75$ and $\vec{v} \approx 0$.

Axisymmetric light field - We next investigate cell motion in light fields with spatially varying polarization. In our experiments, the targeted direction field $\theta_{T}(\vec{r})$ is designed to 
have the form of $\theta_{T}(\vec{r})=k \phi(\vec{r})+\theta_{0}$, where $k$ is a winding number, $\phi=\tan ^{-1}(y / x)$ is the polar angle, and $\theta_{0}$ is a spiral angle (cf. inset of Fig. $2(\mathrm{~b})$ ). When $k=1, \theta_{T}(\vec{r})$ field is axisymmetric as shown by short green lines in Fig. 2 (a-b) and $\theta_{0}$ controls the ratio between bend and splay strength.

Cell motion in axisymmetric fields can be seen in Movies S2-S5 [56]. Quantitatively, mean nematic order parameter, cell velocity, and cell density are plotted in Fig. 2 and Fig. S3 [56]. As shown in Fig. 2(e), nematic order parameter $u$ increases from the defect center to the exterior of the illuminated region, where spatial gradients of $\theta_{T}(\vec{r})$ are small and cells closely follow $\theta_{T}(\vec{r})$. Cells in pure bend $\left(\theta_{0}=\pi / 2\right)$ and mixed $\left(\theta_{0}=3 \pi / 4\right)$ light fields also exhibit mean velocity; peak value in radial profiles in Fig. $2(\mathrm{f})$ is about $50 \mu \mathrm{m} / \mathrm{s}$. Spatial distributions of cells depend on $\theta_{0}$ : while cells aggregate at the exterior boundary for $\theta_{0}=\pi / 2$, Fig. $2(\mathrm{~g})$ shows a relatively flat distribution with a small peak at $r=2.6 \mathrm{~mm}$ for $\theta_{0}=3 \pi / 4$ and cell aggregation near the defect center for other two $\theta_{0}$ conditions. We also systematically vary light intensity and system cell density; qualitatively similar results are shown in Figs. S4-S5 and Movie S7 in the Supplemental Material [56].

Deterministic model - Fig. 1 and Fig. 2 show that cells tend to align their motion direction $\varphi$ towards the local targeted direction $\theta_{T}(\vec{r})$. To quantify this nematic alignment interaction, we extract the time derivative of motion direction $\dot{\varphi}_{j}$ from cell trajectories and find that $\dot{\varphi}_{j}$ is a function of the angular deviation $\varphi_{j}-\theta_{T}\left(\vec{r}_{j}\right)$. We average the dependence function over all cells in a given experiment. Mean $\dot{\varphi}$ in Fig. 3(a) can be adequately described by the following equation:

$$
\dot{\varphi}=-A \sin \left(2\left(\varphi-\theta_{T}\right)\right)+C
$$

Fitting data in Fig. 3(a) leads to a nematic interaction strength $A=0.022 \mathrm{rad} / \mathrm{s}$ [62] and a constant angular velocity $C=-0.005 \mathrm{rad} / \mathrm{s}$ for default light intensity; parameter $A$ increases with light intensity, and $C$ shows a weak dependence, as shown in Fig. S4(e) [56]. Small negative $C$ value indicates that cells have a weak preference to swim clockwise; such chirality has been reported before [36] and is likely caused by the symmetry breaking from handedness of cell body rolling and directionality of the illuminating light, cf. Fig. S1 [56]. This weak chirality explains the non-zero mean cell velocity in an achiral light field in Fig. $2(\mathrm{c})\left(\theta_{0}=\pi / 2\right)$. To describe cell translational motion in our model, we assume all cells have 

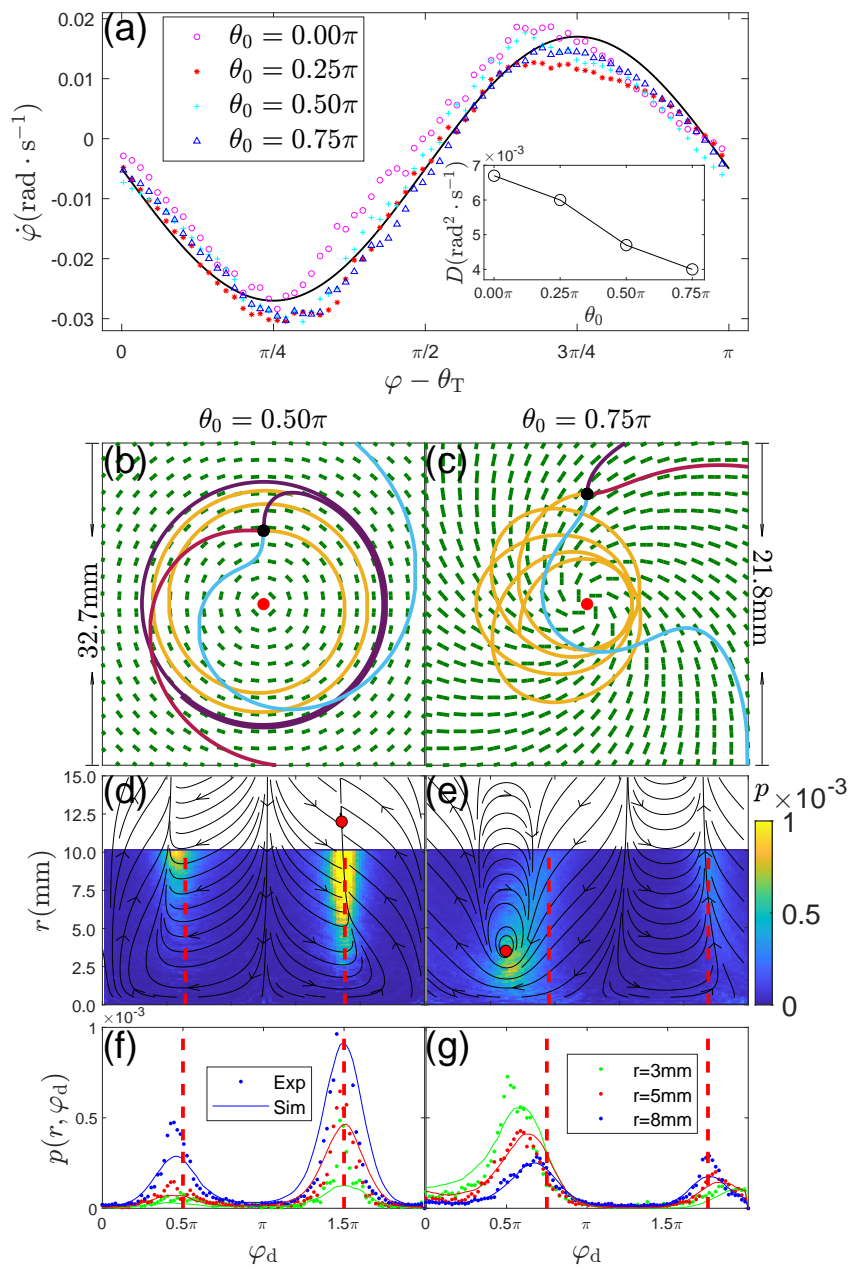

Figure 3. (a) Mean angular velocity $\dot{\varphi}$ versus the angular deviation $\varphi-\theta_{T}$ in in axisymmetric light fields. Inset shows effective diffusivity $D$ measured in different fields $\theta_{0}$. (b-g) Deterministic trajectory and probability distribution in axisymmetric fields with $\theta_{0}=\pi / 2(\mathrm{~b}, \mathrm{~d}, \mathrm{f})$ and $\theta_{0}=3 \pi / 4$ (c, e, g). (b-c) Cell trajectories from the deterministic model plotted on the targeted field. See Fig. S8 [56] for more trajectories. (d-e) Experimentally measured probability $p\left(r, \varphi_{d}\right)$ (color) and computed phase trajectories (black lines). Stable and neutrally stable fixed points are colored in red. Fixed points in $(\mathrm{d})$ is outside of the experimentally measured range $(r<10.8 \mu \mathrm{m})$. (f-g) Profiles of $p\left(r, \varphi_{d}\right)$ at three radii. Dashed lines in $(\mathrm{d}-\mathrm{g})$ mark targeted direction $\theta_{T}$.

the same speed $v_{\circ}=60 \mu \mathrm{m} / \mathrm{s}$ and update cell's position with a velocity

$$
\dot{\vec{r}}=v_{\circ}(\cos \varphi \hat{x}+\sin \varphi \hat{y}) \text {. }
$$

In axisymmetric fields, particle dynamics from Eqs. (1-2) can be described by two variables: the radial coordinate $r$ and the angular deviation from the local polar angle $\varphi_{d}=\varphi-\phi$. 
We solve the governing equations for these quantities (cf. the Supplemental Material [56]) and compute particle trajectories in $\left(r, \varphi_{d}\right)$ phase plane, as shown dark lines in Fig. 3(d-e). Fixed point in the phase plane is identified at $r^{*}=\left|\frac{v_{0}}{C-A \sin 2 \theta_{0}}\right|$ and $\varphi_{d}^{*}=\frac{\pi}{2}\left(\right.$ if $\left.C>A \sin 2 \theta_{0}\right)$ or $\varphi_{d}^{*}=-\frac{\pi}{2}$ (if $C<A \sin 2 \theta_{0}$ ); it is stable if $\cos 2 \theta_{0}<0$, neutrally stable if $\cos 2 \theta_{0}=0$, and unstable if $\cos 2 \theta_{0}>0$. At stable and neutrally stable fixed points, particle moves along circular trajectories, cf. the violet trajectory in Fig. 3(b). Around neutrally stable fixed points, there is a family of closed trajectories in $\left(r, \varphi_{d}\right)$ phase plane; in real space, such trajectories appear to be processing ellipses around the defect center, cf. yellow trajectories in Fig. 3(c) and Fig. S8(c) [56].

Langevin model - Cell motion contains inherent noises, which may arise from flagellum dynamics or cell-cell interactions. To account for this stochasticity, we add a rotational noise term $\sqrt{2 D} \xi(t)$ to Eq. (1), which becomes Eq. (S1) [56]; $\xi(t)$ represents Gaussian white noise with zero-mean $\langle\xi(t) \xi(0)\rangle=\delta(t)$ and $D$ is an effective rotational diffusivity. With this noise term, Eq. (S1) and Eq. (2) constitute a Langevin model of an active Brownian particle whose orientation is locally modulated by the light polarization, i.e. $\theta_{T}$. The corresponding Fokker-Planck equation can be written down for the steady-state probability density, $p(\vec{r}, \varphi)$, of finding a particle at a state $(\vec{r}, \varphi)$. For uniformly polarized field, the probability distribution $p(\varphi)$ can be analytically solved and fitted to data in Fig. 1(c), yielding an estimation of $D / A=0.17 \mathrm{rad}$ for this experiment.

We then consider axisymmetric fields. Probability density $p\left(r, \varphi_{d}\right)$ is experimentally measured and Fig. 3 (d-e) show high value around stable/neutrally stable fixed points. This highlights the importance of fixed points: their radial positions determine cell distributions in Fig. 2(c-d) and they appear at either $\varphi_{d}^{*}=+\frac{\pi}{2}$ or $\varphi_{d}^{*}=-\frac{\pi}{2}$, which breaks the chiral symmetry and leads to a non-zero mean velocity. $p\left(r, \varphi_{d}\right)$ measured in two other cases of $\theta_{0}$ are shown in Fig. S3 [56]. To quantitatively reproduce measured $p\left(r, \varphi_{d}\right)$, we numerically integrate the Langevin model: parameters $A$ and $C$ values extracted from Fig. 3(a) are used and the effective angular diffusivity $D$ is tuned to fit experimental measurements, see inset of Fig. 3(a). Our numerical results agree well with experiments for probability density profiles in Fig. 3 (f-g) and for radial profiles in Fig. 2 (e-g).

Transport of passive particles - Ordered swimming of Euglena cells in Fig. 2 can collectively generate fluid flow [63], which we use hollow glass spheres $(50 \mu \mathrm{m})$ on an air-liquid interface to visualize. Tracer trajectories from an experiment are shown in the top half 


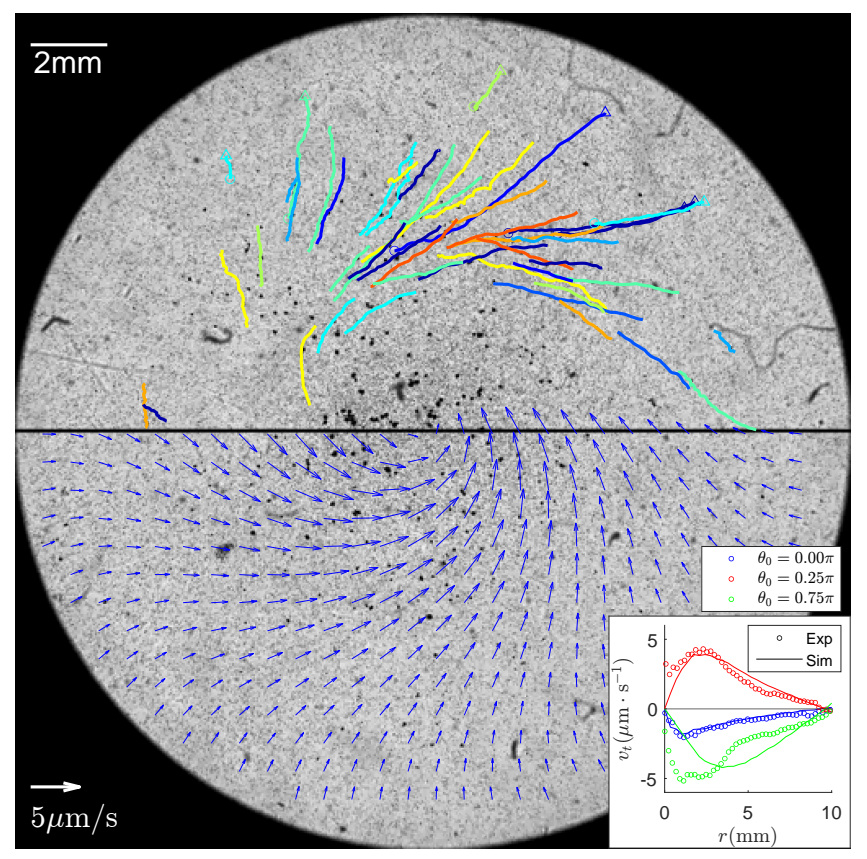

Figure 4. Trajectories of passive tracers (top panel, from experiments) and flow field (bottom panel, from the dipole model) driven by Euglena in a light field with $k=+1$ and $\theta_{0}=\pi / 4$. An experimental snapshot is shown in the background. The inset shows radial profiles of tracers tangential velocities in three axisymmetric $(k=1)$ light fields.

of Fig. 4 and particles spiral counter-clock-wisely towards the center with a peak speed about $5 \mu \mathrm{m} / \mathrm{s}$. To compute the generated flow, we represent swimming cells as force-dipoles [64, 65]: a dipole in a state $(\vec{r}, \varphi)$ generate flow velocity $\vec{w}(\vec{r} ; \vec{r}, \varphi)$ (including contributions from a force-dipole [64] and its image [66, 67]) at a location on the surface $\vec{r}_{s}$. Then, for a given light field, the Langevin model is used to simulate the motion of $N$ cells and to find the probability distribution of cells $p(\vec{r}, \varphi)$. Finally, we compute the total flow as: $\vec{W}\left(\vec{r}_{s}\right)=N \int p(\vec{r}, \varphi) \vec{w}\left(\vec{r}_{s} ; \vec{r}, \varphi\right) \mathrm{d} \vec{r} \mathrm{~d} \varphi$, see Sec. II(F) in the Supplemental Material [56] for details. This approach generates flow fields (cf. bottom half and inset of Fig. 4) that are consistent with measured tracer velocities, see also Fig. S6 [56].

Discussion - Our setup can also generate nonaxisymmetric light fields with integer winding numbers. Fig. 5 shows that cells in a $k=-2$ field form dense and outgoing bands in regions where $\theta_{T}$ is close to be radial; these observations can be explained by stable radial particles trajectories in Fig. S9 (also Movie S6) [56]. The Langevin model is used to investigate light fields with half-integer defects and multiple defects [68]; results of cell 

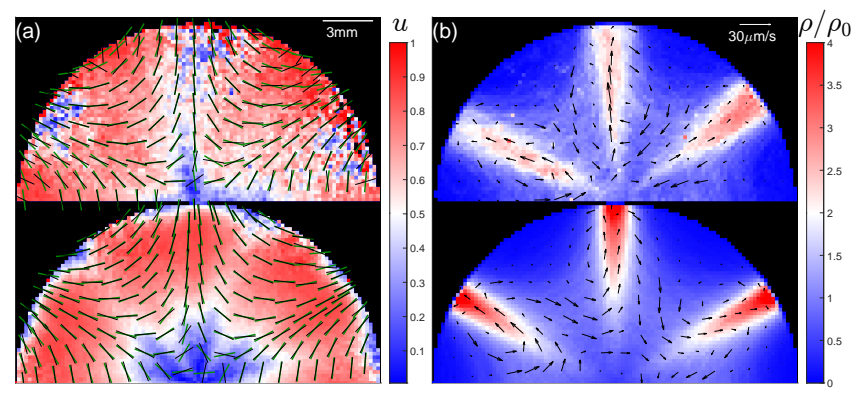

Figure 5. Orientation (a) and velocity/density (b) in a light fields containing a $k=-2$ defect with $\theta_{0}=\pi / 2$. In (a), targeted direction $\theta_{T}$ and mean cell motion direction $\phi_{u}$ are shown by green and black lines, respectively, on nematic order parameter $u$ (in color). In (b), mean cell velocity $\vec{v}$ is plotted on mean density (in color). Top and bottom panels are experimental and numerical results, respectively.

dynamics and transporting flow in Figs. S12 and S13 [56] demonstrate that our idea of local orientation modulation can be used as a versatile and modular method for system control.

Local orientation modulation has been previously implemented by embedding rod-shaped bacteria in nematic liquid crystal with patterned molecular orientation [69 73]. In this bio-composite system, while cell orientation is physically constrained by aligned molecules, bacteria swimming can in return disrupt the molecular order; this strong feedback weakens the controlling ability of the imposed pattern and leads to highly complex dynamics [69 73]. By contrast, our method relies on biological responses, instead of physical interactions, to achieve orientation control, and Euglena motion has no effect on the underlying light field. Such a one-way interaction leads to a much simpler system and may help us to achieve more accurate control. Furthermore, our method works on cells in their natural environment and requires no elaborate sample preparation. This factor and the spatio-temporal tunability of light fields [68] make our method flexible and easy to use.

Sinusoidal term in Eq. (1) is the simplest harmonic for nematic alignment. The same term has been observed in dichroic nano-particle systems [44, 74] and is related to the angular dependence of dichroic light absorption. These nano-particle systems usually require very strong $\left(\sim \mathrm{W} / \mathrm{cm}^{2}-\mathrm{MW} / \mathrm{cm}^{2}\right)$ light stimulus to operate. By contrast, biological response in Euglena greatly amplifies the light signal and functions in the range of $100 \mu \mathrm{W} / \mathrm{cm}^{2}$; this high sensitivity significantly reduces the complexity to construct a controlling light field.

Conclusion - To summarize, we have experimentally demonstrated that Euglena motion 
direction is strongly affected by the local light polarization and that cell dynamics in spatially varying polarization fields is controlled by topological properties and light intensity of the underlying fields. Our experiments also showed that ordered cell swimming, controlled by the polarization field, can generate directed transporting fluid flow. Experimental results have been quantitatively reproduced by an active Brownian particle model in which particle motion direction is nematically coupled to the local light polarization; fixed points and closed trajectories in the model have strong impacts on system properties. These results suggest that local orientation modulation, via polarized light or other means, can be used as a general method to control active matter and micro-scale transporting flow.

Acknowledgments - We acknowledge financial support from National Natural Science Foundation of China (Grants No. 11774222 and No. 11422427) and from the Program for Professor of Special Appointment at Shanghai Institutions of Higher Learning (Grant No. GZ2016004). We thank Hugues Chaté and Masaki Sano for useful discussions and the Student Innovation Center at Shanghai Jiao Tong University for support.

* hepeng_zhang@sjtu.edu.cn

[1] E. Lauga and T. R. Powers, Rep. Prog. Phys. 72, 096601 (2009).

[2] S. Ramaswamy, Annual Review of Condensed Matter Physics 1, 323 (2010).

[3] W. C. K. Poon, Physics of Complex Colloids, ed. C Bechinger, F Sciortino and P Ziherl, 184, 317 (2013).

[4] I. S. Aranson, Phys. Usp. 56, 79 (2013).

[5] W. Wang, W. Duan, S. Ahmed, T. E. Mallouk, and A. Sen, Nano Today 8, 531 (2013).

[6] S. Sanchez, L. Soler, and J. Katuri, Angew. Chem. Int. Ed. 54, 1414 (2015).

[7] J. Elgeti, R. G. Winkler, and G. Gompper, Rep. Prog. Phys. 78, 056601 (50 pp.) (2015).

[8] C. Bechinger, R. Di Leonardo, H. Löwen, C. Reichhardt, and G. Volpe, Giorgio and, Rev. Mod. Phys. 88, 045006 (2016).

[9] O. D. Lavrentovich, Current Opinion in Colloid \& Interface Science 21, 97 (2016).

[10] A. Zottl and H. Stark, J. Phys.: Condens. Matter 28, 253001 (2016).

[11] A. E. Patteson, A. Gopinath, and P. E. Arratia, Current Opinion in Colloid \& Interface Science 21, 86 (2016). 
[12] J. Zhang, E. Luijten, B. A. Grzybowski, and S. Granick, Chem Soc Rev 46, 5551 (2017).

[13] P. Illien, R. Golestanian, and A. Sen, Chem. Soc. Rev. , (2017).

[14] B. Liebchen and H. Loewen, Acc Chem Res 51, 2982 (2018).

[15] G. Gompper, R. G. Winkler, T. Speck, A. Solon, C. Nardini, F. Peruani, H. Lowen, R. Golestanian, U. B. Kaupp, L. Alvarez, T. Kiorboe, E. Lauga, W. C. K. Poon, A. DeSimone, S. MuinosLandin, A. Fischer, N. A. Soker, F. Cichos, R. Kapral, P. Gaspard, M. Ripoll, F. Sagues, A. Doostmohammadi, J. M. Yeomans, I. S. Aranson, C. Bechinger, H. Stark, C. K. Hemelrijk, F. J. Nedelec, T. Sarkar, T. Aryaksama, M. Lacroix, G. Duclos, V. Yashunsky, P. Silberzan, M. Arroyo, and S. Kale, Journal of Physics-condensed Matter 32, 193001 (2020)

[16] J. Wang, Lab. Chip 12, 1944 (2012).

[17] W. Gao and J. Wang, ACS Nano 8, 3170 (2014).

[18] J. X. Li, B. E. F. de Avila, W. Gao, L. F. Zhang, and J. Wang, Science Robotics 2, UNSP eaam6431 (2017).

[19] Y. Alapan, O. Yasa, B. Yigit, I. C. Yasa, P. Erkoc, and M. Sitti, Annual Review of Control, Robotics, and Autonomous Systems, Vol 2 2, 205 (2019).

[20] A. M. Menzel, Physics Reports-review Section of Physics Letters 554, 1 (2015).

[21] H. Stark, European Physical Journal-special Topics 225, 2369 (2016).

[22] M. You, C. Chen, L. Xu, F. Mou, and J. Guan, Acc. Chem. Res. 51, 3006 (2018).

[23] S. Klumpp, C. T. LefĚšvre, M. Bennet, and D. Faivre, Physics Reports 789, 1 (2019).

[24] E. Mikolajczyk, P. L. Walne, and E. Hildebrand, Critical Reviews in Plant Sciences 9, 343 $(1990)$.

[25] G. Jekely, Philosophical Transactions of the Royal Society B-biological Sciences 364, 2795 (2009).

[26] K. Drescher, R. E. Goldstein, and I. Tuval, Proc. Natl. Acad. Sci. U.S.A. 107, 11171 (2010).

[27] L. Barsanti, V. Evangelista, V. Passarelli, A. M. Frassanito, and P. Gualtieri, Integr. Biol. 4, $22(2012)$.

[28] E. A. Kane, M. Gershow, B. Afonso, I. Larderet, M. Klein, A. R. Carter, B. L. de Bivort, S. G. Sprecher, and A. D. T. Samuel, Proc. Natl. Acad. Sci. U.S.A. 110, E3868 (2013).

[29] X. Garcia, S. Rafai, and P. Peyla, Phys. Rev. Lett. 110, 138106 (2013).

[30] A. Giometto, F. Altermatt, A. Maritan, R. Stocker, and A. Rinaldo, Proc. Natl. Acad. Sci. U.S.A. 112, 7045 (2015). 
[31] R. R. Bennett and R. Golestanian, Journal of the Royal Society Interface 12, 20141164 (2015).

[32] R. M. W. Chau, D. Bhaya, and K. C. Huang, Mbio 8, e02330 (2017).

[33] D.-P. Hader and M. Iseki, "Photomovement in euglena," in Euglena: Biochemistry, Cell and Molecular Biology, edited by S. D. Schwartzbach and S. Shigeoka (Springer International Publishing, Cham, 2017) pp. 207-235.

[34] K. Ozasa, J. Won, S. Song, S. Tamaki, T. Ishikawa, and M. Maeda, PLoS One 12, 1 (2017).

[35] J. Arrieta, A. Barreira, M. Chioccioli, M. Polin, and I. Tuval, Sci. Rep. 7, 3447 (2017).

[36] A. C. H. Tsang, A. T. Lam, and I. H. Riedel-Kruse, Nat. Phys. 14, 1216 (2018).

[37] J. Arrieta, M. Polin, R. Saleta-Piersanti, and I. Tuval, Phys. Rev. Lett. 123, 158101 (2019).

[38] S. K. Choudhary, A. Baskaran, and P. Sharma, Biophys. J. 117, 1508 (2019).

[39] L. Xu, F. Mou, H. Gong, M. Luo, and J. Guan, Chem. Soc. Rev. , (2017).

[40] R. Dong, Y. Cai, Y. Yang, W. Gao, and B. Ren, Acc. Chem. Res. 51, 1940 (2018).

[41] J. Wang, Z. Xiong, J. Zheng, X. Zhan, and J. Tang, Acc. Chem. Res. 51, 1957 (2018).

[42] A. Aubret, M. Youssef, S. Sacanna, and J. Palacci, Nat. Phys. (2018).

[43] D. P. Singh, W. E. Uspal, M. N. Popescu, L. G. Wilson, and P. Fischer, Adv. Funct. Mater. 28, 1706660 (2018).

[44] X. Zhan, J. Zheng, Y. Zhao, B. Zhu, R. Cheng, J. Wang, J. Liu, J. Tang, and J. Tang, Adv. Mater. 0, 1903329 (2019).

[45] F. A. Lavergne, H. Wendehenne, T. Bauerle, and C. Bechinger, Science 364, 70 (2019).

[46] J. Arlt, V. A. Martinez, A. Dawson, T. Pilizota, and W. C. K. Poon, Nat. Commun. 9, 768 (2018).

[47] J. Dervaux, M. C. Resta, and P. Brunet, Nat. Phys. 13, 306 (2017).

[48] T. Ogawa, E. Shoji, N. J. Suematsu, H. Nishimori, S. Izumi, A. Awazu, and M. Iima, PLoS One 11, 1 (2016).

[49] J. Stenhammar, R. Wittkowski, D. Marenduzzo, and M. E. Cates, Sci. Adv. 2, (2016),

[50] J. Palacci, S. Sacanna, A. P. Steinberg, D. J. Pine, and P. M. Chaikin, Science 339, 936 (2013).

[51] G. Frangipane, D. Dell'Arciprete, S. Petracchini, C. Maggi, F. Saglimbeni, S. Bianchi, G. Vizsnyiczai, M. L. Bernardini, and R. Di Leonardo, Elife 7, e36608 (2018).

[52] C. Lozano, B. ten Hagen, H. Lowen, and C. Bechinger, Nat. Commun. 7, 12828 (2016).

[53] A. Geiseler, P. Hanggi, F. Marchesoni, C. Mulhern, and S. Savel'ev, Phys. Rev. E 94, 012613 
(2016).

[54] C. CREUTZ and B. O. D. O. DIEHN, The Journal of Protozoology 23, 552 (1976).

[55] D. P. Hader, Arch. Microbiol. 147, 179 (1987).

[56] APS, "See supplemental material at [url] for detailed experimental procedure, additional experimental results, analysis of the langevin model, description of dipole fluid model, and supporting videos." (2020).

[57] M. Rossi, G. Cicconofri, A. Beran, G. Noselli, and A. DeSimone, Proc. Natl. Acad. Sci. U. S. A. 114, 13085 (2017).

[58] N. A. Hill and L. A. PLUMPTON, J. Theor. Biol. 203, 357 (2000).

[59] S. Delaney, M. M. Sanchez-Lopez, I. Moreno, and J. A. Davis, Applied Optics 56, 596 (2017).

[60] H. P. Zhang, A. Be'er, E. L. Florin, and H. L. Swinney, Proc. Natl. Acad. Sci. U. S. A. 107, 13626 (2010).

[61] K. E. BOUND and G. TOLLIN, Nature 216, 1042 (1967).

[62] H. Li, X.-q. Shi, M. Huang, X. Chen, M. Xiao, C. Liu, H. Chate, and H. P. Zhang, Proc Natl Acad Sci USA 116, 777 (2019).

[63] A. J. T. M. Mathijssen, F. Guzman-Lastra, A. Kaiser, and H. Lowen, Phys. Rev. Lett. 121, 248101 (2018).

[64] T. Ogawa, S. Izumi, and M. Iima, J. Phys. Soc. Jpn. 86, 074401 (2017).

[65] D. Bardfalvy, S. Anjum, C. Nardini, A. Morozov, and J. Stenhammar, Physical Review Letters 125, 018003 (2020).

[66] J. Happel and H. Brenner, Low Reynolds Number Hydrodynamics (Prentice Hall, Englewood Cliffs, NJ, 1965).

[67] A. J. T. M. Mathijssen, D. O. Pushkin, and J. M. Yeomans, J. Fluid Mech. 773, 498 (2015).

[68] C. Rosales-Guzman, B. Ndagano, and A. Forbes, Journal of Optics 20, 123001 (2018).

[69] R. R. Trivedi, R. Maeda, N. L. Abbott, S. E. Spagnolie, and D. B. Weibel, Soft Matter 11, 8404 (2015).

[70] C. H. Peng, T. Turiv, Y. B. Guo, Q. H. Wei, and O. D. Lavrentovich, Science 354, 882 (2016).

[71] I. S. Aranson, Acc. Chem. Res. 51, 3023 (2018).

[72] T. Turiv, R. Koizumi, K. Thijssen, M. M. Genkin, H. Yu, C. Peng, Q.-H. Wei, J. M. Yeomans, I. S. Aranson, A. Doostmohammadi, and O. D. Lavrentovich, Nat. Phys. (2020),

[73] R. Koizumi, T. Turiv, M. M. Genkin, R. J. Lastowski, H. Yu, I. Chaganava, Q.-H. Wei, I. S. 
Aranson, and O. D. Lavrentovich, Phys. Rev. Research 2, 033060 (2020).

[74] L. Tong, V. D. Miljkovic, and M. Kall, Nano Lett. 10, 268 (2010). 